\title{
Layer-number determination in graphene on SiC by reflectance mapping
}

\author{
Ivan Gueorguiev Ivanov, Jawad Ul Hassan, Tihomir Iakimov, Alexei A. Zakharov, Rositsa \\ Yakimova and Erik Janzén
}

\section{Linköping University Post Print}

\section{Tweet}

N.B.: When citing this work, cite the original article.

Original Publication:

Ivan Gueorguiev Ivanov, Jawad Ul Hassan, Tihomir Iakimov, Alexei A. Zakharov, Rositsa Yakimova and Erik Janzén, Layer-number determination in graphene on SiC by reflectance mapping, 2014, Carbon, (77), 492-500.

http://dx.doi.org/10.1016/j.carbon.2014.05.054

Copyright: Elsevier

http://www.elsevier.com/

Postprint available at: Linköping University Electronic Press

http://urn.kb.se/resolve?urn=urn:nbn:se:liu:diva-110689 


\title{
Layer-number determination in graphene on $\mathrm{SiC}$ by reflectance mapping
}

Ivan G. Ivanov ${ }^{\text {a, }}$, Jawad Ul Hassan ${ }^{\mathrm{a}}$, Tihomir Iakimov ${ }^{\mathrm{a}}$, Alexei A. Zakharov ${ }^{\mathrm{b}}$, Rositsa Yakimova $^{\mathrm{a}}$, and Erik Janzén ${ }^{\mathrm{a}}$

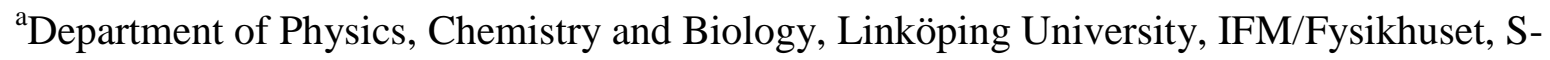
58183 Linköping, Sweden

${ }^{\mathrm{b}}$ MaxLab, Lund University, S-22100 Lund, Sweden

\begin{abstract}
We report a simple, handy and affordable optical approach for precise number-oflayers determination of graphene on $\mathrm{SiC}$ based on monitoring the power of the laser beam reflected from the sample (reflectance mapping) in a slightly modified micro-Raman setup. Reflectance mapping is compatible with simultaneous Raman mapping. We find experimentally that the reflectance of graphene on $\mathrm{SiC}$ normalized to the reflectivity of bare substrate (the contrast) increases linearly with $\sim 1.7 \%$ per layer for up to 12 layers, in agreement with theory. The wavelength dependence of the contrast in the visible is investigated using the concept of ideal fermions and compared with existing experimental data for the optical constants of graphene. We argue also that the observed contrast is insensitive to the doping condition of the sample, as well as to the type of sample (graphene on $\mathrm{C}$ - or $\mathrm{Si}$-face of $4 \mathrm{H}$ or $6 \mathrm{H} \mathrm{SiC}$, hydrogen-intercalated graphene). The possibility to extend the precise layer counting to $\sim 50$ layers makes reflectivity mapping superior to low-energy electron microscopy (limited to 10 layers) in quantitative evaluation of graphene on the Cface of SiC. The method is applicable for graphene on other insulating or semiconducting substrates.
\end{abstract}

\section{Introduction}

A variety of different substrates have been proposed in the past few years aiming at obtaining large-scale graphene sheets for electronic applications. In many cases graphene on semiconductor substrate (conducting or semi-insulating) is ready for device processing with no need for transfer to another substrate, and from this point of view the different polytypes of silicon carbide offer an attractive perspective for large-scale growth by sublimation [1-3] or chemical vapour deposition [4]. In case of sublimation growth on the (0001) Si-face of the hexagonal $4 \mathrm{H}$ or $6 \mathrm{H}$ polytypes of $\mathrm{SiC}$ a so-called buffer layer is formed at the graphene/SiC interface, which is non-conducting and chemically bound to the substrate [5]. It has been

*Corresponding author. Tel: +46 13 282532. E-mail: ivani@ifm.liu.se (I. G. Ivanov). 
shown that hydrogen intercalation can transform the buffer layer into an extra graphene layer; the accompanying decoupling from the substrate results in improved mobility [6]. Single graphene layer can also be produced via H-intercalation, if only a buffer layer is prepared on the $\mathrm{SiC}$ surface prior to the intercalation procedure, as demonstrated previously [7] and in the present article.

The most important feedback parameter to graphene growth on any substrate is the number of layers (or thickness). Atomic force microscopy (AFM) is often quoted as a reliable method applicable to graphene flakes on $\mathrm{SiO}_{2} / \mathrm{Si}$ or other smooth substrates [8], however this method is inappropriate for thickness determination of graphene on $\mathrm{SiC}(\mathrm{G} / \mathrm{SiC})$, because usually all of the surface is covered by single or multiple layers and, in addition, the surface morphology of the substrate is rough enough (due to steps) to mask the contribution from boundaries between graphene areas with different number of layers. It should be noted, however, that phase contrast in AFM is capable of distinguishing buffer layer, mono- and bilayer graphene on $\mathrm{SiC}$ [9], and when AFM is operated in the so-called Kelvin-probe force microscopy mode it allows studying of features in graphene morphology with sub-nanometer resolution [10-11]. Raman mapping has also been considered as a means to distinguish single layer from bilayer and multilayer graphene using the line shape and the position of the so-called 2D Raman peak [12]. While this approach seems reliable in distinguishing one and two from multiple layers for exfoliated graphene (on $\mathrm{SiO}_{2}$ or suspended [13]), in $\mathrm{G} / \mathrm{SiC}$ stress and doping variations occur on microscopic scale and influence the line shape, position and intensity ratio of the Raman lines (G- and 2D-peaks) to a larger extent than the thickness, hence the Raman spectra alone usually do not provide unambiguous layer-number determination [14-15].

Previous work conducted mainly on exfoliated graphene transferred to $\mathrm{SiO}_{2}$ on silicon (graphene/ $\mathrm{SiO}_{2} / \mathrm{Si}$ ) has shown that even single graphene layer is visible in optical microscope if proper oxide thickness and illumination wavelength are selected [16-18]. Blake et al. [16] demonstrate that the wavelength of illumination and the $\mathrm{SiO}_{2}$ thickness have to be matched together for optimal contrast for observation of graphene, because the optical interference in the oxide layer plays a crucial role. Another study [17], also regarding the graphene/ $\mathrm{SiO}_{2} / \mathrm{Si}$ system, suggests using mapping of reflected light from a white-light source and demonstrates distinct contrast spectra (dependence of the contrast on the wavelengths in the white-light source) for up to 10-layer graphene. In both references [16-17] macroscopic theory based on definition of refractive index even for single-layer graphene is used to describe the experimental data. The visibility of graphene on glass in optical microscope without taking 
advantage of any interference contrast enhancement has been also demonstrated in later work [19]; these authors point out also the difficulties with visualizing graphene on high-index substrates (such as $\mathrm{SiC}$ ) due to low contrast.

Concerning $\mathrm{G} / \mathrm{SiC}$, similar purely optical approach for precise quantitative thickness determination in the most interesting region of few-layer graphene is missing, to the best of our knowledge. This is probably due to the low contrast of $\mathrm{G} / \mathrm{SiC}(\sim 1.7 \%$ in reflection and $\sim 1.3 \%$ in transmission) [20-21] compared to $\sim 7 \%$ for graphene on glass [19]. Nevertheless, recent work has succeeded in distinguishing single from bilayer graphene in optical microscope images [22]. However, while this finding is of great importance in evaluating the sample homogeneity, e.g., within devices fabricated on graphene, the optical inspection provides mainly qualitative information on the morphology, not on the exact number of layers within the different areas visible in the microscope. Attempts to use absorption $[21,23]$ or attenuation of the Raman signal from the substrate [24] for thickness determination of G/SiC have only had partial success, since the error margin is acceptable only for a large number of layers, a case of no big practical interest. The absorption method used in [23] suffers insufficient spatial resolution, but recently proposed micro-transmission imaging [25] overcomes this limitation, offering the microscopic resolution necessary for investigating the morphology of G/SiC. The authors [25] use micro-transmission in combination with Raman spectroscopy to investigate the properties of mono- and bilayer $\mathrm{G} / \mathrm{SiC}$. However, as mentioned above the contrast in absorption (transmission) is somewhat lower than that in reflection (contrary to suspended graphene), and the back-side quality as well as the dopingdependent transparency of the SiC substrate may influence the transmitted intensity. Thus, so far the most reliable method of thickness determination for few-layer G/SiC is considered to be the low-energy electron microscopy (LEEM) [26], which allows also imaging. However, this method is not very convenient for routine characterization, since it is rather expensive and requires preparation procedures prior to experiment. We note also that thickness determination by LEEM is restricted to about 10 layers, while more than hundred layers are often observed on the C-face of SiC. Thus, LEEM has restricted capabilities for routine characterization of graphene grown intentionally on the C-face. Another method making use of the contrast in scanning electron microscopy [27] suffers similar restrictions.

Thus, a handy, quantitative, purely optical method capable of evaluating the exact number of layers and imaging graphene morphology with sub-micrometer spatial resolution is highly desirable. We propose here an approach based on mapping the power of the laser beam 
reflected from the sample (reflectance mapping) in a most common micro-Raman setup. The method provides quick imaging of areas comparable with those usually observed in LEEM. In our particular samples, we have observed all the reflectance values associated with up to 12 layers $\mathrm{G} / \mathrm{SiC}$, but extension to up to 50 layers is possible, as indicated by the theoretical consideration, which provides the possibility to study the thickness morphology also for graphene on the $\mathrm{C}$-face of $\mathrm{SiC}$. An important advantage is the possibility of simultaneous Raman and reflectance mapping, which provides valuable independent information on the thickness in the interpretation of the Raman spectra.

\section{Results and discussion}

\subsection{Experimental details}

The experimental setup is outlined schematically in Fig. 1. The main modification to an otherwise conventional micro-Raman spectrometer is the addition of two beam splitters (BS1 and BS2). In fact, when infinity-corrected objective is used, the laser light reflected from the sample travels exactly the same way but backwards with respect to the incoming laser beam. BS1 separates the reflected laser beam from the incident one and directs the former to BS2. The purpose of BS2 is to divide the reflected beam into two counterparts, one of which is used to control the focus via a CCD camera, while the power of the other is measured using a power meter. Reference intensity is readily obtained by recording the power reflected from a bare $\mathrm{SiC}$ substrate, usually averaged over a number of points. Care should be taken, however, to ensure oxide-free reference substrate, see the Supplementary Data (Section S1) for details. We utilize autofocusing of the beam on the sample during mapping using the image of the laser spot from the CCD camera as a feedback for precise vertical positioning of the sample, which is mounted on a piezo positioner (not shown). The latter is mounted in its turn on a computer-driven X-Y table (not shown) used for scanning the sample under the beam during mapping. Thus, the maximum lateral resolution can be maintained during the whole mapping procedure. We have estimated the laser spot diameter on the sample to about $400 \mathrm{~nm}$ (full diameter at half-maximum intensity) with our objective of magnification 100, which means that the corresponding size of the Airy disk is about $800 \mathrm{~nm}$. Another optional power meter can be used as shown in Fig. 1 for recording the slow-term variations of the laser power; this reference data can be used in processing the map for renormalization of the measured reflectance from the sample and/or the bare substrate.

The samples used in this work were prepared by high-temperature sublimation of $4 \mathrm{H}$ and $6 \mathrm{H}$ $\mathrm{SiC}$ in a broad temperature interval $\left(1400-2000{ }^{\circ} \mathrm{C}\right)$ and include samples grown in vacuum, 
as well as in Ar ambient with pressure in the range 50 - 1000 mbar. Further details on the growth conditions and the hydrogen intercalation are presented elsewhere [2-3].

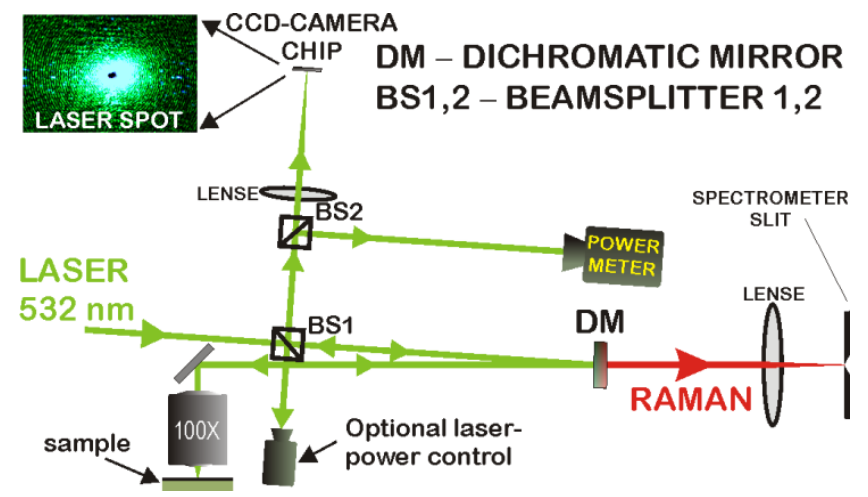

Fig. 1 - Schematic presentation of the experimental setup illustrating the separation of the incident and the reflected beams, and the compatibility with simultaneous Raman measurement.

\subsection{Experimental results}

We will demonstrate now that the intensity of the laser beam reflected from the sample contains unambiguous information on the number of graphene layers. Initially, we investigated a sample containing only monolayer (light areas) and islands of bilayer graphene (dark areas), as established by the LEEM images shown in Fig. 2(a) for two different areas on the same sample. The variation of the reflected laser intensity vs. point number on part of a square map on this sample is depicted in Fig. 2(b). As seen in the figure, the reflected beam intensity from the graphene sample varies essentially between two values, which are separated by the same amount as the lower value is separated from the value for bare $\mathrm{SiC}$, i.e., approximately $1.7 \%$. Values in-between the former two are easily understood as originating from partial coverage of one- and two-layer areas by the laser spot. In Fig. 2(c), a reflectance map measured on the same sample and in the same scale as the LEEM images is displayed by colour-coding each intensity value. The similarity with the morphology displayed in the LEEM images of Fig. 2(a) is obvious, hinting at the conclusion, from purely experimental point of view, that the higher reflectance value corresponds to bilayer (islands), while the lower value represents the reflectivity of single graphene layer [continuous area, cf. Fig. 2(a)]. Note that each layer contributes by about the same amount in the reflected intensity, i.e. $1.7 \%$ of the reference intensity (denoted $R_{\mathrm{SiC}}$ in the figure). 

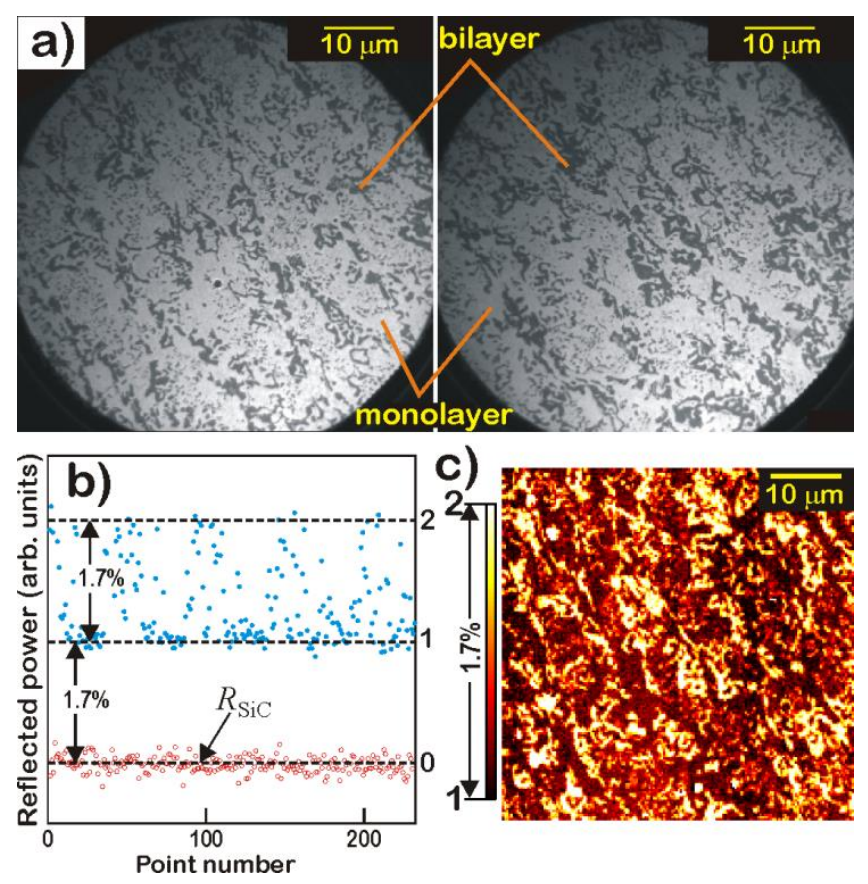

Fig. 2 - Results from a sublimation-grown graphene on $4 \mathrm{H} \mathrm{SiC:} \mathrm{a)} \mathrm{LEEM} \mathrm{images} \mathrm{of} \mathrm{two}$ different areas on the sample, confirming presence of only monolayer and bilayer graphene. b) Variation of the reflected laser intensity for different points in a map on the same sample (closed circles) compared to that of reference bare substrate (open circles). The horizontal dashed lines are drawn at the levels $R_{\mathrm{SiC}}(1+0.017 \mathrm{~N})$, where $\boldsymbol{R}_{\mathrm{SiC}}$ denotes the average reflected power from the substrate, and the values of $N=0,1,2$ are denoted to the right of each line. (c) Reflectance map on the same sample drawn in the same scale as the LEEM image in part (a). The colours in the colour bar span the region between the levels $N=1$ and $N=2$.

The investigation of other samples shows that the increase in reflected intensity by $1.7 \%$ per layer holds also for more than two layers. Since graphene grown on the $\mathrm{C}$-face of a $\mathrm{SiC}$ substrate is known to exhibit large variety in the number of layers, this kind of samples are suitable for testing the reflectance of multiple layers. Fig. 3 illustrates the variation of the reflected laser power from samples grown on the $\mathrm{C}$-face of $4 \mathrm{H}$ or $6 \mathrm{H} \mathrm{SiC}$ substrates normalized to the average power reflected from bare substrate. The data represents parts of several millimetres-long linear scans on different C-face samples allowing us to observe multiple plateaus in the reflectance corresponding to different numbers of layers. Similar to Fig. 2, the horizontal dotted lines in Fig. 3 correspond to the values $(1+0.017 N)$ with the values of $N$ displayed to the right of the plot. The reference power used to normalize the data is obtained by averaging a few hundred experimental points on the substrate (one set of such points is displayed along the line $N=0$ ). We notice that reflectivity of many different substrates of the $4 \mathrm{H}$ and $6 \mathrm{H}$ polytypes of $\mathrm{SiC}$ was measured, both on $\mathrm{C}$ - and the Si-faces, but any differences are well within the experimental error and could not be identified. This is 
expected in view of the same (within the experimental error) refractive index of $4 \mathrm{H}$ and $6 \mathrm{H}$ SiC. As seen in Fig. 3, different plateaus occur as a rule at intensities $R_{\mathrm{SiC}}(1+0.017 N)$, hence $N$ can be attributed to the number of layers, allowing us to establish the empirical relation

$R(N)=R_{\mathrm{SiC}}(1+0.017 N)$,

where $R(N)$ denotes the reflectance of $N$ layers of G/SiC. The most important observation from the data presented in Fig. 3 and other similar data (not shown) is that we are able to identify plateaus related to reflection from up to 12 graphene layers with no significant deviation from the linear dependence implied by Eq. (1). On the other hand, it is obvious that the linear dependence of Eq. (1) must start saturating for large enough number of layers, and in the limit $N \rightarrow \infty$ the reflectance $R(N)$ should tend to the reflectivity of graphite. However, for $N \leq 12$ any non-linearity in the dependence $R(N)$ seems to be within the experimental error. Although we certainly encounter regions with $N>20$ or more in our samples and plateaus are still visible for $N>12$ (not shown), we have not identified all the plateaus up to these values of $N$. Hence, their association with definite number of layers requires further statistics and may lead to detection of the expected bowing in $R(N)$.

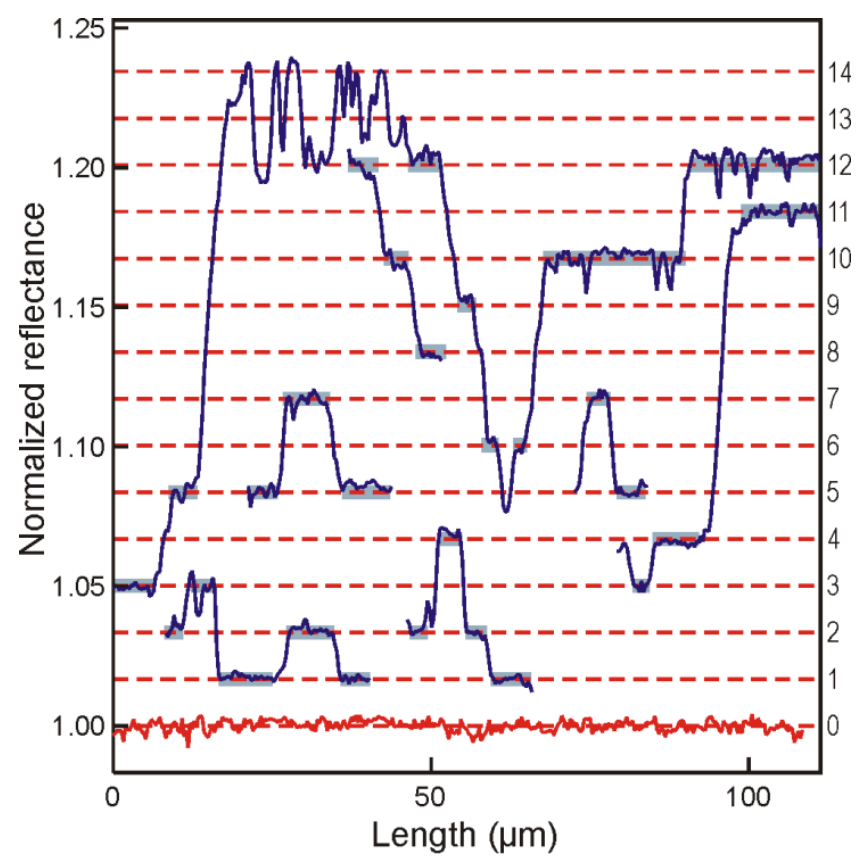

Fig. 3 - Normalized reflectance traces obtained by linear scans on different samples grown by sublimation on the $\mathrm{C}$-face of $4 \mathrm{H}$ or $6 \mathrm{H} \mathrm{SiC}$ illustrating identification of plateaus (outlined) corresponding to up to $N=12$ graphene layers. All data is normalized to the average reflectivity of bare substrate (one set of data points [bottom curve] corresponding to zero layers, i.e., bare substrate is also shown). Similarly to Fig. 2, 


\section{the horizontal dashed lines correspond to the levels $(1+0.017 N)$ with the values of $N$ denoted to the right. The horizontal axis represents a common scale for the length of the traces shown; the step in each trace is $300 \mathbf{~ n m}$.}

We will argue later that the factor 0.017 in Eq. (1) should not actually depend (at least, not strongly) on experimental factors, such as numerical aperture and working distance of the objective used, neither should it exhibit strong dependence on the laser wavelength. For the time being we discuss the application of our method to different species of G/SiC, such as graphene on $\mathrm{Si}$ - or $\mathrm{C}$-face, and hydrogen-intercalated graphene. Fig. 4 shows examples of reflectance maps for different types of samples, illustrating the applicability of the method in all cases. In processing the maps for graphene on $\mathrm{C}$ and $\mathrm{Si}$ faces, we did not notice any significant deviation from Eq. (1), despite the different properties of these species concerning the presence or absence of buffer layer, as well as the stacking sequence in case of few-layer graphene (FLG). On Si-face, there exists a buffer layer and FLG forms in Bernal stacking sequence, while on the C-face there is no buffer layer and the layers in FLG are rotationally misoriented [5], which leads to decoupling of the layers. (We note that different structure of graphene on C-face SiC consisting in rotationally-misoriented domains of Bernal-stacked graphene has also been reported [28].) Neither the stacking arrangement nor the presence of buffer layer seems to influence the reflectance results, which are generally in agreement with Eq. (1). Similar observation has been made for multiple H-intercalated graphene samples, with one exception concerning the monolayer graphene regions of the $\mathrm{H}$-intercalated sample presented in Fig. 4(f). Prior to intercalation, this particular sample had large areas on the surface covered with buffer layer only, which has been lifted into a single graphene layer after $\mathrm{H}$-intercalation (the upper right part of the map in Fig. 4(f) displays several such areas). In this particular sample, and only on the monolayer areas, we have observed a gradient in the reflectance, which is easily seen as change in the colour across the area, e.g., in the area marked as a single layer with "1" in Fig. 4(f). No such gradient is observed in the bilayer areas or in any of the other $\mathrm{H}$-intercalated samples, which typically had minimum two layers [cf. Fig. 4(e)]. The origin of this graded reflectance is not understood at present. We note that both the buffer layer and the hydrogen atomic layer in the case of $\mathrm{H}$-intercalation are essentially transparent and non-conductive, therefore their influence on the reflectance is expected to be negligible. 
a) $4 \mathrm{H}$, Si-face

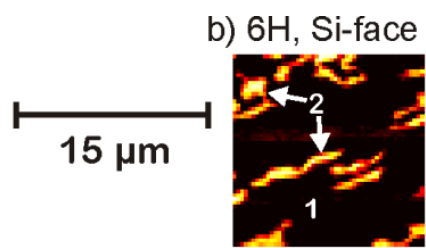

c) $4 \mathrm{H}$, Si-face, $2-3$ layers

d) $4 \mathrm{H}, \mathrm{C}$-face
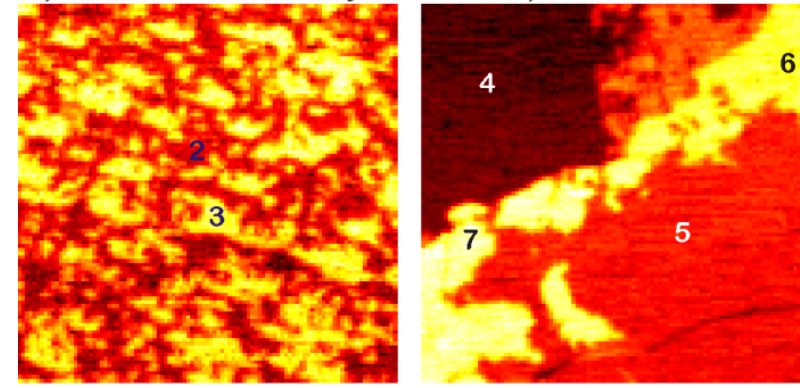

e) $4 \mathrm{H}, \mathrm{H}$-intercalated

f) $4 \mathrm{H}, \mathrm{H}$-intercalated minimum 2 layers

with monolayer
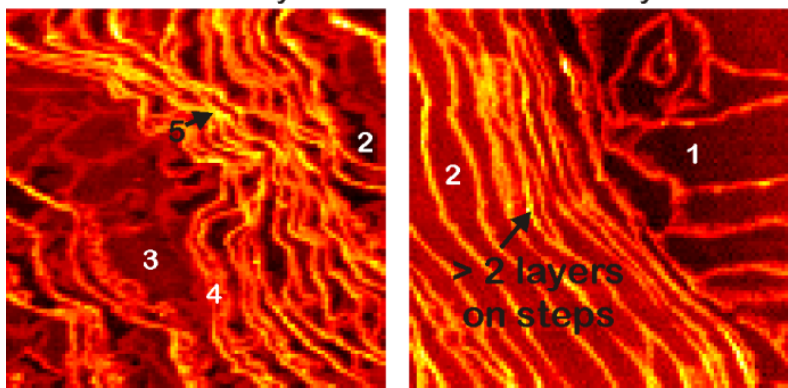

Fig. 4 - Examples of reflectance maps on a variety of graphene samples on SiC: a), b) sublimation-grown samples on the Si-face of $4 \mathrm{H}$ and $6 \mathrm{H} \mathrm{SiC}$, respectively; c) thicker sample on the Si-face of $4 \mathrm{H} \mathrm{SiC} \mathrm{obtained} \mathrm{with} \mathrm{prolonged} \mathrm{growth} \mathrm{time;} \mathrm{d)} \mathrm{sample} \mathrm{grown}$ on the $\mathrm{C}$-face of $\mathbf{4 H ~ S i C}$; e), f) $\mathrm{H}$-intercalated samples grown on the Si-face. The sample in part (f) shows large areas of monolayer graphene, whereas the minimum number of layers in the other $\mathrm{H}$-intercalated sample (e) is two. The number of layers determined in accord with Eq. (1) is denoted in one of the areas in each map. The scale bar (15 $\mu \mathrm{m})$ and the step used $(300 \mathrm{~nm})$ are common for all maps.

\subsection{Comparison with theory}

We turn now to discussion of the experimental results and the appearance of Eq. (1) in the light of the existing theory. Recent theoretical work [20,29] treats the conductivity of graphene within the tight binding approximation and provides analytical expressions for the optical conductivity, which is in close relation to the reflectivity. In addition to the calculation of the conductivity, Stauber et al [20] derive also the formulas describing transmission and reflectivity of a single graphene layer separating two media with relative dielectric permittivities $\varepsilon_{1}$ and $\varepsilon_{2}$. In the case of $\mathrm{G} / \mathrm{SiC}$ the first media is air (or vacuum), hence $\varepsilon_{1}=1$, and the second media is $\mathrm{SiC}$ with refractive index $n_{S i C}=\sqrt{\varepsilon_{2}} \approx 2.55[21,23]$. Then the 
equation for the reflectivity $R(1)$ in the case of a single graphene layer on $\mathrm{SiC}$ (Eq. (52) of [20]) becomes

$$
R(1)=\frac{\left|n_{S i C}+\sigma(\omega) /\left(\varepsilon_{0} c\right)-1\right|^{2}}{\left|n_{S i C}+\sigma(\omega) /\left(\varepsilon_{0} c\right)+1\right|^{2}}
$$

Here $\varepsilon_{0}$ denotes the dielectric permittivity of vacuum, $c$ is the speed of light, and $\sigma(\omega)$ is the conductivity of the graphene layer. Note that while $n_{S i C}$ is real in the visible part of the spectrum, $\sigma(\omega)$ may be complex, hence the modulus sign in the numerator and denominator. However, it has been shown previously [30-31] that as long as optical frequencies are considered, $\sigma(\omega)$ can be replaced by the universal conductivity $\sigma_{0}=\frac{e^{2}}{4 \hbar}(e$ denotes the electron charge); the neglect of the imaginary part will be further justified later. Consequently, $\sigma(\omega) /\left(\varepsilon_{0} c\right) \approx \pi \alpha$, where $\alpha \cong 1 / 137$ is the fine structure constant. In this approximation, the generalization of Eq. (2) for small number of graphene layers $\mathrm{N}$ becomes

$$
R(N) \cong \frac{\left|n_{S i C}+N \pi \alpha-1\right|^{2}}{\left|n_{S i C}+N \pi \alpha+1\right|^{2}} \cong R_{S i C}\left(1+\frac{4 \pi \alpha}{n_{S i C}^{2}-1} N\right)
$$

Here $R_{S i C}=\left(\frac{n_{S i C}-1}{n_{S i C}+1}\right)^{2}$ is the reflectivity of bare SiC. The last approximate equality in Eq. (3) justifies the appearance of the empirical Eq. (1) and is valid for $N \ll<\frac{n_{S i C}^{2}-1}{\pi \alpha}$, i.e., $N \ll 67$. In generalizing Eq. (2) to the case of FLG $(N>1)$ we make use of the commonly accepted concept that the conductivity of $\mathrm{N}$ graphene layers is essentially a sum of the conductivities of the separate layers. Most importantly, the prefactor $4 \pi \alpha /\left(n_{S i C}^{2}-1\right) \approx 0.0167$ is very close to the empirical factor 0.017 in Eq. (1). This is in spite of the fact that the above equations are valid for normal incidence only, while our microscope objective (numerical aperture NA = 0.95) collects light reflected at angles up to $\sim 70^{\circ}$. Thus, integration over the spatial angle corresponding to the NA of the objective is sometimes proposed for obtaining accurate results [19], but the agreement between theory and experiment suggests that such integration is not essential in our case and would only slightly modify the theoretical factor of 0.0167 . This can be seen from the following argument. The laser has well defined polarization, hence light reflected under a certain angle will contain equal contributions from $s$ - and $p$-polarizations. 
However, when absorption is weak (as in our case), the general appearance of the reflectivity curves for $s$ - and $p$-polarized light is such that their average approximately equals the reflectivity value at normal incidence for angles well below the Brewster angle. This is not an accurate approximation for large angles of incidence (around and above the Brewster angle), but such angles have only minor contribution in the total reflection due to the Gaussian distribution of the laser intensity in the beam cross-section. Weak dependence on the numerical aperture of the objective has been reported also for graphene on glass substrates $(<$ $0.04 \%$ ) [19] and on bulk $\mathrm{SiO}_{2}$ substrate [31]. Thus, we neglect the integration procedure.

Equation (3) offers a simple and accurate expression for the contrast of FLG ( $\operatorname{small} N$ ) on any transparent (for the wavelengths of interest) substrate. From the common definition of contrast $[8,17]$ (in this case, contrast in reflection) we obtain for the contrast of FLG

$$
C(N)=\frac{R(N)-R_{S i C}}{R_{S i C}} \cong \frac{4 \pi \alpha}{n_{S i C}^{2}-1} N .
$$

Similar expression can be found in Ref. [31]. The contrast is lower for graphene on higher index substrate, which stipulates that the approximate linear change of contrast with the number of layers according to Eq. (4) is valid for larger $N$ compared to graphene on low-index substrate, e.g., glass or $\mathrm{SiO}_{2}$. In particular, the contrast of $\sim 1.7 \%$ for single layer on $\mathrm{SiC}$ (refractive index $\sim 2.55$ ) is by a factor of four to five lower than the contrast on glass (typical refractive indices in the range $1.46-1.55$ ), which might explain the non-trivial efforts succeeding only recently in distinguishing single-layer from bilayer graphene on $\mathrm{SiC}$ in optical microscope [22], whereas graphene on glass or $\mathrm{SiO}_{2}$ has been routinely observed in microscope for years.

Note that the general form of Eq. (3) is actually not valid for large number of layers, because for $N \rightarrow \infty, R(N) \rightarrow 1$ (the appropriate limit would be the reflectivity of graphite). It is interesting therefore to compare Eq. (3) to an equation, which adopts macroscopic approach to FLG on a substrate by assigning complex refractive index to graphene, as is done, for instance, in the work of $\mathrm{Ni}$ et al [17]. In this work the authors consider the trilayer system graphene $/ \mathrm{SiO}_{2} / \mathrm{Si}$; in our case we have to deal with the bilayer system graphene/SiC and, following similar approach, we assign a complex refractive index $n_{1}=n+i \kappa$ to the graphene layer(s). The equation describing the reflectance of $\mathrm{G} / \mathrm{SiC}$ then becomes [32] 
$R(d)=\left|\frac{r_{1} e^{i \beta}+r_{2} e^{-i \beta}}{e^{i \beta}+r_{1} r_{2} e^{-i \beta}}\right|^{2}$,

where the phase difference $\beta$ depends on the thickness $d$ of graphene, $\beta=\beta(d)=2 \pi n_{1} d / \lambda, \lambda$ is the wavelength, and $r_{1}=\frac{n_{0}-n_{1}}{n_{0}+n_{1}}, r_{2}=\frac{n_{1}-n_{2}}{n_{1}+n_{2}}$ are the reflection coefficients at the interfaces air/graphene $\left(n_{0}=1\right)$ and graphene/SiC $\left(n_{2} \equiv n_{S i C} \approx 2.55\right)$. The thickness is expressed as $d=N d_{0}$, where $d_{0}=0.335 \mathrm{~nm}$ is the "thickness" of a single layer (the layer separation in graphite). We are interested in the expression for $R(d)$ in the limit $d \rightarrow 0$, which corresponds to small number of layers $N$ and can be directly compared to Eq. (3). Thus, in the limit $d / \lambda<<1$ one obtains in a straightforward manner

$$
R(d) \approx R_{S i C}\left(1+\frac{4}{n_{S i C}^{2}-1} 4 \pi n \kappa \frac{d}{\lambda}\right)=R_{S i C}\left(1+\frac{4}{n_{S i C}^{2}-1} 4 \pi n \kappa \frac{d_{0}}{\lambda} N\right)
$$

Using the textbook relations

$$
n^{2}-\kappa^{2}=\operatorname{Re} \varepsilon(\omega), \text { and } 2 n \kappa=\operatorname{Im} \varepsilon(\omega)=\frac{\sigma(\omega)}{\varepsilon_{0} c}
$$

where $\varepsilon(\omega)$ is the dielectric function, it is not difficult to see that Eq. (6) reduces to Eq. (3) after replacing $\sigma(\omega)$ with $\sigma_{0}$, as expected because both equations are deduced on the ground of Fresnel equations. However, now we dispose of a general expression, Eq. (5), which is valid for all values of $d$ and correctly tends to the reflectivity of graphite $\left|\left(n_{0}-n_{1}\right) /\left(n_{0}+n_{1}\right)\right|^{2}$ when $d \rightarrow \infty$. We note also that the real part of the dielectric function, which is related to the imaginary part of the conductivity does not appear at all in the expression to the first order of $d / \lambda$ (it does appear in the second order). Similarly, if instead of replacing $\sigma(\omega)$ with $\sigma_{0}$ we use the complex expression for $\sigma(\omega)$, e.g., from Ref. [29] we will find that the imaginary part of $\sigma(\omega)$ is involved only in terms quadratic with respect to the product $\pi \alpha$, which justifies their neglect for FLG. Furthermore, the comparison of Eqs. (6) and (3) yields the following relation

$4 \pi n \kappa d_{0} / \lambda=\pi \alpha$ 
Ni et al [17] use constant values $n=2.0$ and $\kappa=1.1$ to fit their data in the whole visible region of the spectrum, but constant values are incompatible with Eq. (8). In later work of Bruna and Borini [33] we find essentially the same expression as Eq. (8) [their Eq. (2)] deduced on the ground of the definition of extinction coefficient and the observed absorption of $\pi \alpha$ in single graphene layer by Nair et al [34]. The only difference is that in their formula $-\ln (1-\pi \alpha)$ appears instead of $\pi \alpha$ as in ours, but the discrepancy is insignificant being of order of $(\pi \alpha)^{2}$. The authors [33] propose the use of constant $n=3.0$ and $\kappa=C \lambda$, where $C$ is a constant estimated to $1.82 \mu^{-1}$ in this work. With these values they are able to describe significant amount of data obtained within the whole spectral region between $\sim 0.5$ and $3 \mathrm{eV}$ and extending from FLG to graphite. Adopting the value $n=3.0$ and adjusting $\kappa=1.794 \lambda$ (if $d_{0}=0.335 \mathrm{~nm}$ instead of $0.34 \mathrm{~nm}$ as in Bruna and Borini is used, $C \cong 1.794$ is obtained), we may use the exact form, Eq. (5), instead of Eq. (3) for calculating the reflectance. The dependence of the reflectance of $\mathrm{G} / \mathrm{SiC}$ on the number of layers for the wavelength $\lambda=532$ $\mathrm{nm}=0.532 \mu \mathrm{m}$ used in our experiments is calculated using Eqs. (3) and (5) and displayed in Fig. 5 (thick solid line) together with the linear approximation (thin solid line in the insert) common for the two expressions. The difference between the curves and the straight line is quite insignificant as long as several (less than $~ 20$ ) layers are considered, as illustrated in the insert of Fig. 5. We note that the increasing discrepancy between Eqs. (3) and (5) for $N>\sim 40$ is merely a consequence of neglect of any interference effects in Eq. (3), hence Eq. (5) is the relevant one to be used for larger number of layers. Fig. 5 suggests also that with proper calibration reflectance mapping can be used for determination of up to $\sim 50$ graphene layers owing to the non-vanishing derivative of the reflectance vs. number-of-layers dependence.

Although all measurements in this work are performed using laser wavelength of $532 \mathrm{~nm}$ $(2.33 \mathrm{eV})$, we may still discuss the anticipated wavelength dependence of the contrast (reflectance) on the ground of existing data for the refractive index and extinction coefficient of graphene and graphite [35-36]. To this end we have discussed the reflectance in the approximation when the electronic band dispersion in graphene is that of non-interacting fermions, i.e., conical dispersion near the $K$-points of the Brillouin zone. This allows us to replace the real conductivity of graphene $\sigma(\omega)$ with the universal conductivity $\sigma_{0}$. As long as this approximation is valid, the slope of the linear dependence valid in the case of FLG $(N<$ 20) depends on the wavelength $\lambda$ only weakly through the dispersion of the refractive index of the substrate, $n_{S i C}=n_{S i C}(\lambda)$, according to Eqs. (3), (6) and (8). It is appropriate to notice 
here that this anticipated weak dependence of the single-layer contrast on the wavelength is drastically different from the strong wavelength dependence observed for graphene/SiO $/ 2 \mathrm{Si}$ [37]. Indeed, in this work [37] it is explained that the role of the $\mathrm{SiO}_{2}$ layer is to act as a spacer, changing the phase of the light reflected from silicon, hence the strong wavelength dependence allowing tailoring the contrast for specific wavelength by varying the thickness of the $\mathrm{SiO}_{2}$ layer. On the contrary, interference effects in the case of $\mathrm{G} / \mathrm{SiC}$ start playing significant role only when graphene thickness becomes sufficient to induce interference between the beams reflected from the two interfaces, $\mathrm{G} / \mathrm{SiC}$ and air/graphene. In the case of few-layer graphene interference effects are clearly negligible, cf. Fig. 5.

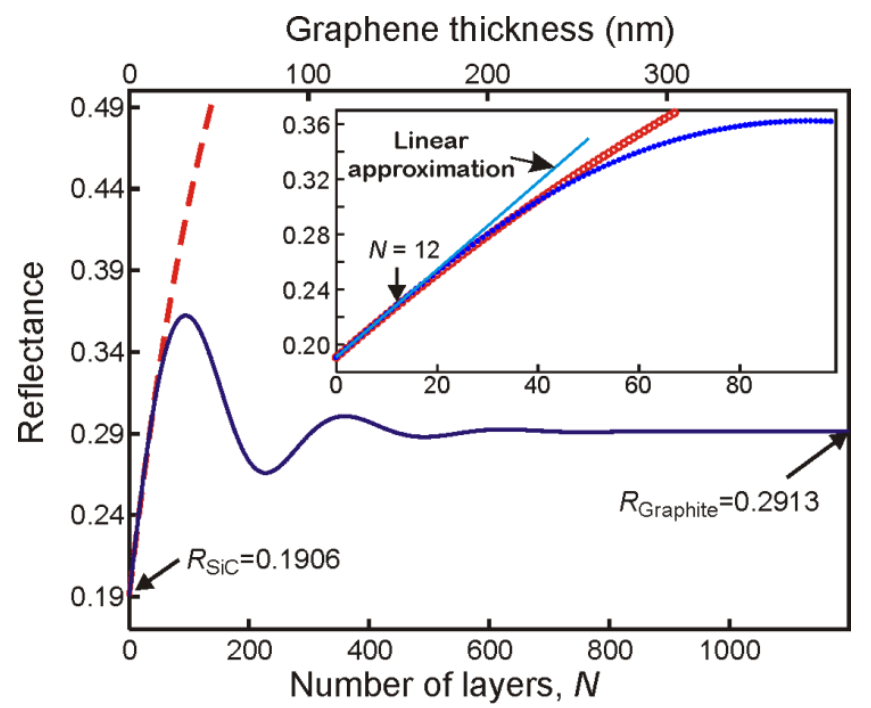

Fig. 5 - Variation of the reflectance of graphene on $\mathrm{SiC}$ with its thickness expressed in number of layers $N$, according to Eq. (3) [dashed line] and (5) [solid line]. The plot is valid for wavelength $\lambda=0.532 \mu \mathrm{m}, n=3.0$, and $\kappa=1.794 \lambda=0.9544$. At $N \geq \sim 800$ the reflectivity is practically indistinguishable from that of graphite. The insert zooms into the initial part of the curves (the case of FLG) and displays also the linear approximation [cf. Eq. (3)], which is common for both dependencies.

The breakdown of the Dirac-fermions approximation for higher photon energies might have more pronounced impact on the observed contrast than the dispersion of the substrate refractive index, which for transparent materials (e.g., $4 \mathrm{H}-\mathrm{SiC}$ ) usually does not exceed $3-4 \%$ of the refractive index value across the visible range. The breakdown of the Dirac-fermions approximation at higher energies, mainly due to triangular warping of the electronic energy bands of graphene away from the Dirac point, results in deviation of the conductivity $\sigma(\omega)$ from the universal conductivity $\sigma_{0}$. Theoretical calculations with account for triangular 
warping predict increase of the ratio $\sigma(\omega) / \sigma_{0}$ from $\sim 1.005$ to $\sim 1.04$ in the range of photon energies $1-3 \mathrm{eV}$ (see, e.g., [20] and the Supplementary information of Ref. [34]). However, for energies below $2.33 \mathrm{eV}$ (corresponding to wavelength of $532 \mathrm{~nm}$ used in this work) the deviation of $\sigma(\omega) / \sigma_{0}$ from one does not exceed $2 \%$, according to theory. We already saw that $\sigma(\omega)$ is related to the product $n \kappa$ [cf. Eq. (7)], hence an increased value of $\sigma(\omega)$ above $\sigma_{0}$ will be expressed in increased product $n \kappa$ above the linear dependence on the wavelength $\lambda$ given by Eq. (8). The refractive index $n$ and extinction coefficient $\kappa$ for graphene on $\mathrm{Si}, \mathrm{SiO}_{2} / \mathrm{Si}$ or quartz substrates have been measured independently in two different works using spectroscopic ellipsometry [35-36]. In Fig. 6 we compare the data for the product $n \kappa$ acquired from the figures in these references with the linear dependence of Eq. (8), which corresponds to ideal non-interacting Dirac fermions. As expected, all experimental points lie above the theoretical straight line. Both sets of experimental points asymptotically approach the theoretical line at lower energies (higher wavelengths) and the disagreement becomes insignificant as a consequence of more accurate fulfilment of the Dirac-fermions approximation. However, not only the discrepancy between the (ideal) theory and experiment, but also the discrepancy between the two experimental data sets increases rapidly towards higher photon energies. For example, around $530 \mathrm{~nm}$, the data points of Ref. [35] are by 26\% higher than the theoretical value, while the disagreement between Eq. (8) and the data of Ref. [36] is $\sim 12 \%$. We notice that if solely the increase of $\sigma(\omega) / \sigma_{0}$ to $\sim 1.02$ (around $530 \mathrm{~nm}$ ) is accounted for $[20,34]$ the expected increase in $n \kappa$ above the linear dependence, Eq. (8), would be only $\sim 2 \%$. Furthermore, the absorption of single layer graphene also increases with increasing $\sigma(\omega) / \sigma_{0}$. The data of Ref. [34] suggests that all data points for the absorption of single layer graphene and for wavelengths above $500 \mathrm{~nm}$ lie around the value $\pi \alpha \cong(2.3 \pm$ $0.1) \%$, i.e., within an error margin of $\pm 5 \%$. The data of Refs. [35] and [36] would predict absorption of single layer graphene around $530 \mathrm{~nm}$ by $26 \%$ and $12 \%$ larger than $\pi \alpha$, respectively, which is not confirmed experimentally. Our contrast in reflectance of $1.7 \%$ is also in better agreement with the concept of ideal fermions utilised in the work of Bruna and Borini [33]. Indeed, using the values from [35] and [36] at $\sim 532 \mathrm{~nm}$ for the product $n \kappa$ would yield a contrast of $\sim 2.1 \%$ and $\sim 1.9 \%$ at normal incidence, respectively, instead of the observed $1.7 \%$. This difference cannot be accounted for by integrating over the numerical aperture of the objective, because such integration only tends to increase (albeit insignificantly, as already discussed) the true value for normal incidence; thus the $1.7 \%$ observed in our work is the approximate upper bound of the true value for the contrast at 
normal incidence. Similarly, good agreement with the optical constants proposed by Bruna and Borini [33] has been observed in Ref. [19] for reflectance of graphene on glass substrate. The wavelength used in this work is close to ours, $\sim 550 \mathrm{~nm}$ selected with bandpass filter. (We recall that actually only the product $n \kappa$ is of importance for reflectance measurements on few-layer graphene, not the individual values of $n$ and $\kappa$ ). We notice that sample contamination has been discussed previously as possible reason for increased absorption at higher photon energies [34], and has been shown to affect significantly also ellipsometric measurements [38], hence it might be at least partially responsible for the outlined discrepancy of absorption and reflectance measurements with ellipsometric data. However, in the energy range above $\sim 2.5 \mathrm{eV}$ (wavelengths below $\sim 500 \mathrm{~nm}$ ) one may expect growing contribution from the wing of the absorption peak at $4.6 \mathrm{eV}$ [35-36], which is probably the reason for the inflection seen in Fig. 6 in both experimental data sets around $500 \mathrm{~nm}$. This contribution will possibly lead to slight increase of the contrast (from $1.7 \%$ at $530 \mathrm{~nm}$ to at most $\sim 3 \%$ at $400 \mathrm{~nm}$, judging from Fig. 6). Thus, no drastic changes are expected in the contrast within the whole visible region (more details on the wavelength dependence of the contrast are discussed in Sec. S2 of the Supplementary Data).

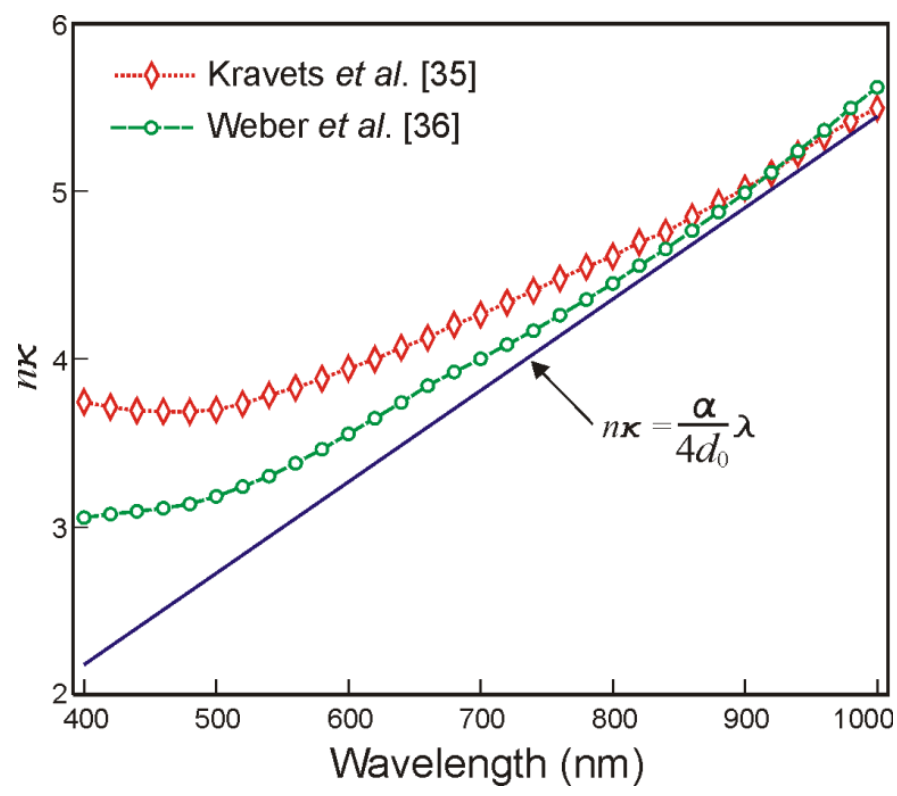

Fig. 6 - Comparison of the dependence of the product $n \kappa$ vs. wavelength calculated in the approximation of ideal Dirac fermions [33] (straight line) with the experimental values from Refs. [35] (diamonds) and [36] (circles).

Our experimental data suggests also that the increase of reflectance by $\sim 1.7 \%$ per layer is rather insensitive to the position of the Fermi level (chemical potential) $\mu$ with respect to the 
electro-neutrality point. This observation can be justified using the complex expression for the conductivity, e. g., of Ref. [29]; the chemical potential appears in the imaginary part of the interband conductivity (which is the dominating one at optical frequencies) through the expression [29]

$$
\operatorname{Im} \sigma(\omega)=\frac{\sigma_{0}}{2 \pi} \ln \frac{(\hbar \omega+2 \mu)^{2}}{(\hbar \omega-2 \mu)^{2}+(2 k T)^{2}}
$$

Here $k$ is the Boltzmann constant and $T$ the temperature. For optical frequencies and room temperature, $\hbar \omega>>k T$ and the thermal-energy term in the denominator of the logarithm can be neglected. Also, the chemical potential for G/SiC usually does not exceed $0.2-0.4 \mathrm{eV}$ [39], so that $\hbar \omega>>\mu$. Thus, for optical frequencies $\operatorname{Im} \sigma(\omega) /\left(\varepsilon_{0} c\right)$, which is involved in the reflectivity of graphene [cf. Eq.(2)] can be quite accurately approximated with the expression

$$
\operatorname{Im} \sigma(\omega) /\left(\varepsilon_{0} c\right) \approx \frac{4}{\pi} \frac{\mu}{\hbar \omega} \pi \alpha
$$

This expression appears squared, e.g., when the squared modulus of the numerator and the denominator of Eq. (2) is evaluated, and it is clearly negligible compared to the other terms, not only because of the smallness of $(\pi \alpha)^{2}$, but also due to the small factor $(\mu / \hbar \omega)^{2}$. Thus, we obtain yet another justification for neglecting the imaginary part of $\sigma(\omega)$ for FLG. This leads us to conclude also that variations of the chemical potential $\mu$ have negligible effect on reflectivity (cf. also Fig. 6 of Stauber et al [20]) and are not a likely reason for the observed gradient in the reflectivity of the single-layer regions of the $\mathrm{H}$-intercalated sample mentioned earlier, see Fig. 4(f).

\section{Conclusions}

We have successfully employed mapping of the reflected intensity of the exciting laser in a slightly modified micro-Raman setup in order to visualize the morphology in terms of exact number of layers of graphene grown on SiC. The reflectance mapping alone is much faster than Raman mapping; a map containing 10000 points can be obtained in less than two hours, but only information on the number of layers is obtained. However, reflectance mapping can be performed also simultaneously with Raman mapping, thus providing valuable information on the number of graphene layers in addition to the information about strain and doping obtainable from the Raman spectra. We find also excellent agreement between the experimental data and existing theory. Simple expressions describing the reflectance of FLG 
are proposed, and the quantum mechanical results are compared with a macroscopic approach, in which graphene is described with refractive index and extinction coefficient. This comparison leads to anticipation that in principle up to $\sim 50$ graphene layers can be accurately determined, making the technique more suitable alternative than LEEM for investigation of graphene on the $\mathrm{C}$-face. The reflectance-mapping method is applicable for counting the graphene layers also on other semiconducting or insulating substrates.

\section{Acknowledgments}

Support from GRAPHIC-RF and EPIGRAT projects within the EuroGRAPHENE program of the European Science Foundation (ESF) is gratefully acknowledged.

\section{References}

[1] Berger C, Song Z, Li T, Li X, Ogbazghi AY, Feng R, et al. Ultrathin epitaxial graphite: $2 \mathrm{D}$ electron gas properties and a route toward graphene-based nanoelectronics. J Phys Chem B 2004;108(52):19912-6.

[2] Tzalenchuk A, Lara-Avila S, Kalaboukhov A, Paolillo S, Syvävärvi M, Yakimova R, et al. Towards a quantum resistance standard based on epitaxial graphene. Nature Nanotech 2010;5(3):186-9.

[3] Hassan J, Virojanadara C, Meyer A, Ivanov IG, Flege JI, Watcharinyanon S, et al. Control of epitaxial graphene thickness on $4 \mathrm{H}-\mathrm{SiC}(0001)$ and buffer layer removal through hydrogen intercalation. Mat Sci Forum 2012;717-720:605-8.

[4] Strupinski W, Grodecki K, Wysmolek A, Stepniewski R, Szkopek T, Gaskell PE, et al. Graphene epitaxy by chemical vapor deposition on SiC. Nano Lett 2011;11(4):1786-91.

[5] Hass J, De Heer WA, Conrad EH. The growth and morphology of epitaxial multilayer graphene. J Phys: Condens Matter 2008;20(32):323202.

[6] Robinson JA, Hollander M, Labella M III, Trumbull KA, Cavalero R, Snyder DW. Epitaxial graphene transistors: Enhancing performance via hydrogen intercalation. Nano Lett 2011;11(9):3875-80.

[7] Speck F, Jobst J, Fromm F, Ostler M, Waldmann D, Hundhausen M, et al. The quasifree-standing nature of graphene on H-saturated SiC(0001). Appl Phys Lett 2011;99(12):122106.

[8] Ni ZH, Wang Y, Yu T, Shen Z. Raman spectroscopy and imaging of graphene. Nano Res 2008;1(4):273-91. 
[9] Bolen ML, Harrison SE, Biedermann LB, Capano MA. Graphene formation mechanisms on 4H-SiC(0001). Phys. Rev. B 2009;80(11):115433.

[10] Filleter T, Emtsev KV, Seyller T, Bennewitz R. Local work function measurements of epitaxial graphene. Appl. Phys. Lett. 2008;93(13):133117.

[11] Eriksson J, Pearce R, Iakimov T, Virojanadara C, Gogova D, Andersson M, et al. The influence of substrate morphology on thickness uniformity and unintentional doping of epitaxial graphene on SiC. Appl. Phys. Lett. 2012;100(24):241607.

[12] Malard LM, Pimenta MA, Dresselhaus G, Dresselhaus MS. Raman spectroscopy in graphene. Phys Rep 2009;473(5-6):51-87.

[13] Ferrari AC, Meyer JC, Scardaci V, Casiraghi C, Lazzeri M, Mauri F, et al. Raman spectrum of graphene and graphene layers. Phys Rev Lett 2006;97(18):187401.

[14] Grodecki K, Bozek R, Strupinski W, Wysmolek A, Stepniewski R, Baranowski JM. Micro-Raman spectroscopy of graphene grown on stepped 4H-SiC (0001) surface. Appl Phys Lett 2012;100(26):261604.

[15] Robinson JA, Puls CP, Staley NE, Stitt JP, Fanton MA, Emtsev KV, et al. Raman topography and strain uniformity of large-area epitaxial graphene. Nano Lett 2009;9(3):964-8.

[16] Blake P, Hill EW, Castro Neto AH, Novoselov KS, Jiang D, Yang R, et al. Making graphene visible. Appl Phys Lett 2007;91(6):063124.

[17] Ni ZH, Wang HM, Kasim J, Fan HM, Yu T, Wu YH, et al. Graphene thickness determination using reflection and contrast spectroscopy. Nano Lett 2007;7(9):2758-63.

[18] Nolen CM, Denina G, Teweldebrhan D, Bhanu B, Balandin AA. High-throughput largearea automated identification and quality control of graphene and few-layer graphene films. ACS Nano 2011;5(2):914-22.

[19] Gaskell PE, Skulason HS, Rodenchuk C, Szkopek T. Counting graphene layers on glass via optical reflection microscopy. Appl Phys Lett 2009;94(14):143101.

[20] Stauber T, Peres NMR, Geim AK. Optical conductivity of graphene in the visible region of the spectrum. Phys Rev B 2008;78(8):085432.

[21] Dawlaty JM, Shivaraman S, Strait J, George P, Chandrashekar MVS, Rana F, et al. Measurement of the optical absorption spectra of epitaxial graphene from $\mathrm{THz}$ to visible. Appl Phys Lett 2008;93(13):131905.

[22] Yager T, Lartsev A, Mahashabde S, Charpentier S, Davidovikj D, Danilov A, et al. Express optical analysis of epitaxial graphene on $\mathrm{SiC}$ : Impact of morphology on quantum transport. Nano Lett 2013;13(9):4217-23. 
[23] Drabinska A, Grodecki K, Strupinski W, Bozek R, Korona KP, Wysmolek A, et al. Growth kinetics of epitaxial graphene on $\mathrm{SiC}$ substrates. Phys Rev $\mathrm{B}$ 2010;81(24):245410.

[24] Shivaraman S, Chandrashekar MVS, Boeckl JJ, Spencer MG. Thickness estimation of epitaxial graphene on $\mathrm{SiC}$ using attenuation of substrate Raman intensity. J Electron Mater 2009;38(6):725-30.

[25] Tiberj A, Camara N, Godignon P, Camassel J. Micro-Raman and micro-transmission imaging of epitaxial graphene grown on the $\mathrm{Si}$ and $\mathrm{C}$ faces of $6 \mathrm{H}-\mathrm{SiC}$. Nanoscale Research Letters 2011;6:478.

[26] Hibino H, Kageshima H, Maeda F, Nagase M, Kobayashi Y, Yamaguchi H. Microscopic thickness determination of thin graphite films formed on $\mathrm{SiC}$ from quantized oscillation in reflectivity of low-energy electrons. Phys Rev B 2008;77(7):075413.

[27] Park MH, Kim TH, Yang CW. Thickness contrast of few-layered graphene in SEM. Surf Interface Anal 2012;44(11-12):1538-41.

[28] Johansson LI, Watcharinyanon S, Zakharov AA, Iakimov T, Yakimova R, Virojanadara C. Stacking of adjacent graphene layers grown on C-face SiC. Phys Rev B 2011;84(12):125405.

[29] Falkovsky LA, Pershoguba SS. Optical far-infrared properties of a graphene monolayer and multilayer. Phys Rev B 2007;76(15):153410.

[30] Kuzmenko AB, Van Heumen E, Carbone F, Van der Marel D. Universal optical conductance of graphite. Phys Rev Lett 2008;100(11):117401.

[31] Mak KF, Sfeir MY, Wu Y, Lui CH, Misewich JA, Heinz TF. Measurement of the optical conductivity of graphene. Phys Rev Lett 2008;101(19):196405.

[32] Anders H. Thin films in optics. London and New York: The Focal Press; 1967.

[33] Bruna M, Borini S. Optical constants of graphene layers in the visible range. Appl Phys Lett 2009;94(3):031901.

[34] Nair RR, Blake P, Grigorenko AN, Novoselov KS, Booth TJ, Stauber T, et al. Fine structure constant defines visual transparency of graphene. Science 2008;320(5881):1308.

[35] Kravets VG, Grigorenko AN, Nair RR, Blake P, Anissimova S, Novoselov KS, Geim AK. Spectroscopic ellipsometry of graphene and an exciton-shifted van Hove peak in absorption. Phys. Rev. B 2010;81(15):155413. 
[36] Weber JW, Calado VE, van den Sanden MCM. Optical constants of graphene measured by spectroscopic ellipsometry. Appl. Phys. Lett. 2010;97(9):091904.

[37] Casiraghi C, Hartschuh A, Lidorikis E, Qian H, Harutyunyan H, Gokus T, et al. Rayleigh imaging of graphene and graphene layers. Nano Lett. 2007;7(9):2711-7.

[38] Jellison GE, Jr., Hunn JD, Lee HN. Measurements of optical functions of highly oriented pyrolytic graphite in the visible. Phys. Rev. B 2007;76(8):085125.

[39] Riedl C, Coletti C, Starke U. Structural and electronic properties of epitaxial graphene on $\mathrm{SiC}(0001)$ : A review of growth, characterization, transfer doping and hydrogen intercalation. J. Phys. D: Appl. Phys. 2010;43(1):1-17. 
